Bundesgesundheitsbl 2012 $55: 308-317$

DOI 10.1007/s00103-011-1430-y

Online publiziert: 25. Februar 2012

(c) Springer-Verlag 2012

D. Laußmann · M. Haftenberger · U. Langen · D. Eis

Abteilung Epidemiologie und Gesundheitsberichterstattung, Fachgebiet Epidemiologie nicht übertragbarer Erkrankungen, Robert Koch-Institut, Berlin

\title{
Einflussfaktoren für Asthma bronchiale bei Kindern und Jugendlichen in Deutschland
}

\section{Ergebnisse der KiGGS-Studie}

\section{Hintergrund}

Asthma bronchiale ist eine chronisch entzündliche Erkrankung der Atemwege, die durch eine reversible Obstruktion der Atemwege mit variabler Ausprägung und eine erhöhte Reizbarkeit der Atemwege (Hyperreagibilität) charakterisiert wird. Symptome, die häufig im Zusammenhang mit Asthma bronchiale auftreten, sind das anfallsweise Auftreten von Atemnot, pfeifende Atemgeräusche und Hustenattacken $[1,2]$.

Bei Kindern und Jugendlichen ist Asthma bronchiale eine häufig auftretende Erkrankung. In der „International Study of Asthma and Allergies in Childhood I (ISAAC I) “ lag die Asthmaprävalenz in den frühen 1990er-Jahren bei Kindern aus den 155 Untersuchungszentren in 55 Ländern bei 10,2\% für die Sechs- bis Siebenjährigen und bei $11,3 \%$ für die 13 bis 14-Jährigen, wobei erhebliche Unterschiede zwischen verschiedenen Ländern beobachtet wurden [3]. In den deutschen ISAAC-Zentren Münster und Greifswald lag die Lebenszeitprävalenz damals im Durchschnitt bei 3,6\% für die Sechs- bis Siebenjährigen und bei 5,7\% für die 13 bis 14-Jährigen [4]. In den westlichen Ländern hat die Prävalenz von Asthma bronchiale und Asthmasymptomen bis in die 1990er-Jahre bei Kindern und jungen Erwachsenen kontinuierlich zugenommen. Ab Mitte der 1990er-Jahre nahm die Asthmaprävalenz in vielen Ländern weiter zu.
In einigen Ländern ist jedoch eine Stagnation oder sogar eine Abnahme der Asthmaprävalenz zu verzeichnen $[5,6]$.

Für die betroffenen Kinder und Jugendlichen ist eine Erkrankung an Asthma bronchiale mit körperlichen Einschränkungen und seelischen Belastungen verbunden [7]. Auch die volkswirtschaftlichen Folgen sind beträchtlich. Beispielsweise lagen im Jahr 1999 die Kosten für die Behandlung eines Asthmapatienten im Kindes- beziehungsweise Jugendalter je nach Schweregrad zwischen 2200 und 7900 Euro pro Jahr [8].

Die Entstehung von Asthma bronchiale ist komplex und bis heute nicht vollständig geklärt. Es findet sich eine Interaktion von endogenen (genetischen) Faktoren und zahlreichen exogenen Faktoren wie Umweltbelastungen, Lebensstil- und sozialen Faktoren [9].

Obwohl Einflussfaktoren für Asthma bronchiale bereits in bestimmten Studienpopulationen auf regionaler Ebene in Deutschland untersucht wurden, liegen bisher keine bundesweiten Studien bei Kindern und Jugendlichen vor. Ziel der vorliegenden Auswertung war es, anhand der bundesweit erhobenen Daten des deutschen Kinder- und Jugendgesundheitssurveys (KiGGS) Einflussfaktoren zu identifizieren, die mit dem Auftreten von Asthma bronchiale bei Kindern und Jugendlichen assoziiert sind. Dabei wird insbesondere der Frage nachgegangen, ob im KiGGS erhobene soziale Faktoren, In- nenraum- und Außenluftbelastungen sowie der Atopiestatus und die genetische Prädisposition der Kinder und Jugendlichen mit einer gegenwärtig bestehenden Asthmaerkrankung in Verbindung stehen.

\section{Methoden}

Der Kinder- und Jugendgesundheitssurvey (KiGGS) ist eine bundesweite, repräsentative Studie zum Gesundheitszustand von Kindern und Jugendlichen, die von Mai 2003 bis Mai 2006 vom Robert Koch-Institut (RKI) durchgeführt wurde. Konzept, Design und Durchführung des KiGGS wurden bereits an anderen Stellen ausführlich beschrieben $[10,11]$. Die Zielpopulation des KiGGS umfasste Kinder und Jugendliche im Alter von null bis 17 Jahren, die mit ihrem Hauptwohnsitz in der Bundesrepublik Deutschland gemeldet waren. Insgesamt wurden 28.299 Kinder und Jugendliche durch Stichprobeziehung aus den Melderegistern von 167 für Deutschland repräsentativen Städten und Gemeinden ausgewählt und zur Untersuchung eingeladen. Davon nahmen 17.641 Heranwachsende (8985 Jungen, 8656 Mädchen) zusammen mit ihren Eltern teil. Die Teilnahmequote lag bei $66,6 \%$. Untersuchungen zur Non-Response und zur Repräsentativität unter Berücksichtigung der NonResponder wurden durchgeführt und an anderer Stelle publiziert [12]. Die Kinder und deren Eltern/Bezugspersonen wur- 


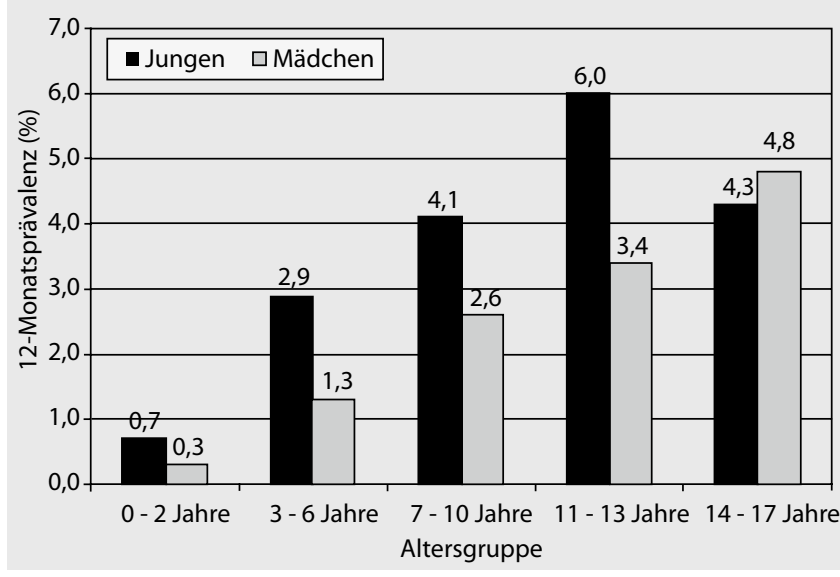

Abb. $1<$ Zwölf-Monats-Prävalenz von Asthma bronchiale, geschichtet nach Alter und Geschlecht (KiGGS, gewichtet) den über die Studie und alle mit der Studie zusammenhängenden Untersuchungen aufgeklärt. Der KiGGS wurde durch den Bundesbeauftragten für Datenschutz und die Ethikkommission des Virchowklinikums der Berliner Humboldt-Universität begutachtet $[10,11]$.

Die Datenerfassung erfolgte durch altersspezifische standardisierte Fragebögen für Eltern/Bezugspersonen (aller Kinder) und parallel durch Fragebögen für Kinder ab elf Jahre. Darüber hinaus fanden eine medizinische Untersuchung des Kindes inklusive Blutabnahme für Laboranalysen und eine standardisierte ärztliche Befragung (computergestütztes ärztliches Interview, CAPI) des begleitenden Elternteils beziehungsweise der Bezugsperson statt.

Informationen zu Asthma bronchiale wurden bei der ärztlichen Befragung (CAPI) erhoben. Die Fragen lauteten: „Hat ein Arzt jemals bei Ihrem Kind Asthma festgestellt?" und „Ist die Krankheit in den letzten zwölf Monaten aufgetreten?“. Zudem wurden der Zeitpunkt des erstmaligen Auftretens der Erkrankung und die Anwendung von Asthmamedikamenten in den letzten zwölf Monaten erfragt. In der vorliegenden Publikation wurde eine Asthmaerkrankung angenommen, sofern im CAPI angegeben wurde, dass die Krankheit in den letzten zwölf Monaten aufgetreten war und/oder in diesem Zeitraum eine spezifische Medikamenteneinnahme berichtet wurde.

Die Auswahl möglicher Einflussfaktoren bezüglich der Zwölf-Monats-Prävalenz von Asthma bronchiale stützt sich auf die Ergebnisse vorausgegangener Studien beziehungsweise auf die ein- schlägige Literatur. Daten zur Soziodemografie der Probanden wie Alter, Geschlecht, sozialer Status, Migrationshintergrund, Wohnort in Ost-/Westdeutschland, Beruf der Eltern, Gemeindegröße, Geschwisterzahl sowie Art und Beginn der Kinderbetreuung wurden den Fragebögen entnommen. Angaben zu frühkindlichen Einflüssen wie das Alter der Mutter bei der Geburt, das Rauchverhalten der Mutter in der Schwangerschaft, das Stillverhalten der Mutter und das Geburtsgewicht stammten ebenfalls aus dieser Datenquelle. Die familiäre Vorbelastung wurde anhand der Frage nach dem Vorliegen einer atopischen Erkrankung (Asthma, Neurodermitis oder Heuschnupfen) bei einem der leiblichen Elternteile erfasst. Daten über Innenraumbelastungen wie Rauchen der Eltern in der Wohnung, schimmlige Wände im Wohnbereich des Kindes und Haustierhaltung wurden auch aus den Fragebogenangaben entnommen. Informationen zum eigenen Rauchverhalten und zum Aufenthalt in verrauchten Räumen waren nur für Kinder ab elf Jahre verfügbar und beruhten auf Selbstangaben der Kinder in den Fragebögen. Das Lebensalter wurde in fünf Klassen (null bis zwei Jahre, drei bis sechs Jahre, sieben bis zehn Jahre, elf bis 13 Jahre und 14 bis 17 Jahre) eingeteilt. Auf Basis von Einkommen, Berufsstatus und Schulbildung der Eltern wurde der soziale Status nach Winkler [13] gebildet und in die Kategorien niedrig, mittel und hoch eingeteilt. Ein Kind hatte einen Migrationshintergrund, wenn es selbst aus einem anderen Land zugezogen war und mindestens ein Elternteil nicht die deutsche Staatsange- hörigkeit hatte (a) oder wenn beide Eltern nicht in Deutschland geboren wurden oder keine deutsche Staatsangehörigkeit hatten (b). Der Body-Mass-Index (BMI) wurde aus der Körpermasse, dividiert durch das Quadrat der Körperlänge, berechnet und in $\mathrm{kg} / \mathrm{m}^{2}$ angegeben. Eine Klassifikation nach dem BMI wurde auf Basis der Referenzwerte von KromeyerHauschild et al. vorgenommen [14].

Weitere allergische/atopische Vorerkrankungen (Neurodermitis und Heuschnupfen) beim Kind wurden dem ärztlichen Interview (CAPI) entnommen. Für beide Erkrankungsarten wurde erfragt, ob diese jemals von einem Arzt diagnostiziert worden waren, ob sie in den letzten zwölf Monaten aufgetreten waren und in welchem Alter das Kind erstmalig erkrankt war. In der vorliegenden Auswertung war es von besonderem Interesse zu prüfen, ob das zeitlich vorangehende Auftreten von Neurodermitis oder Heuschnupfen ein Einflussfaktor für Asthma bronchiale war. Eine Vorerkrankung an Heuschnupfen oder Neurodermitis lag vor, wenn das Datum der Erstdiagnose dieser Erkrankungen zeitlich vor dem der Asthmadiagnose lag. Keine Vorerkrankung bestand, wenn die Diagnosen von Asthma bronchiale und Heuschnupfen oder Neurodermitis zeitgleich gestellt wurden.

Die statistischen Analysen wurden mit dem Statistikprogramm SPSS Version 14 durchgeführt. Um die Clusterstruktur des Studiendesigns zu berücksichtigen, erfolgten alle Auswertungen mit dem SPSS-Verfahren für komplexe Stichproben unter Verwendung eines Stichproben-Gewichtungsfaktors, der die Abweichung der Stichprobe von der Bevölkerungsstruktur (Stand: 31.12.2004) korrigiert. Im ersten Schritt wurden die Prävalenzen einer (in den letzten zwölf Monaten) bestehenden Asthmaerkrankung, geschichtet nach den potenziellen Einflussgrößen, ermittelt und mit dem Chi-Quadrat-Test auf Signifikanz geprüft. Mittels multivariater logistischer Regressionsanalyse wurden danach die unabhängigen Einflussfaktoren für das Auftreten von Asthma in den letzten zwölf Monaten bei Kindern und Jugendlichen ermittelt. 


\section{Ergebnisse}

\section{Alter und Geschlecht}

Im KiGGS wurde bei 4,7\% der Kinder und Jugendlichen jemals Asthma bronchiale diagnostiziert. Von 3,3\% der Nullbis 17-Jährigen wurde in den letzten zwölf Monaten vor der Untersuchung über mindestens eine Asthmaepisode und/oder eine entsprechende Medikamentenanwendung berichtet. Die ZwölfMonats-Prävalenz nahm mit dem Alter $\mathrm{zu}$ (• Abb. 1). Bis zur Altersgruppe elf bis 13 Jahre waren Jungen häufiger betroffen als Mädchen. In der Altergruppe der 14- bis 17-Jährigen kehrt sich das Bild um, hier war der Anteil der Mädchen mit Asthma bronchiale etwas höher als der der Jungen.

\section{Sozioökonomische Faktoren}

Die Asthmaprävalenz in den zwölf Monaten vor der Befragung unterschied sich nicht nach sozialem Status, dem Migrationshintergrund oder der Wohnregion (Ost/West) (• Tab. 1). Heranwachsende, die in einer ländlichen oder kleinstädtischen Umgebung wohnten, hatten jedoch signifikant seltener Asthma bronchiale als Heranwachsende, die in Großstädten aufwuchsen. Bei Kindern mit mindestens einem Elternteil, das von Beruf Landwirt war, war die Asthmaprävalenz geringer, jedoch nicht signifikant geringer als bei Kindern, deren Eltern keine Landwirte waren. Die Asthmaprävalenz bei Kindern mit zwei oder mehr älteren Geschwistern war signifikant geringer als bei Kindern ohne ältere Geschwister. Eine direkte Korrelation zwischen der Asthmaprävalenz und der Anzahl der Geschwister fand sich jedoch nicht. Bei Kindern, die bis zum Schulalter ausschließlich in der Familie betreut wurden, war die ZwölfMonats-Prävalenz von Asthma bronchiale etwas niedriger als bei Kindern, die im Vorschulalter in einer Kindereinrichtung betreut wurden. Bei den Kindern, die in einer Kindereinrichtung betreut wurden, hatte das Alter beim Betreuungsbeginn keinen Einfluss auf die Asthmaprävalenz.

Bundesgesundheitsbl 2012 ·55:308-317 DOI 10.1007/s00103-011-1430-y

(c) Springer-Verlag 2012

\section{Laußmann · M. Haftenberger · U. Langen · D. Eis}

\section{Einflussfaktoren für Asthma bronchiale bei Kindern und Jugendlichen in Deutschland. Ergebnisse der KiGGS-Studie}

\section{Zusammenfassung}

Im vorliegenden Beitrag wurden Zusammenhänge zwischen einer bestehenden Asthmaerkrankung und möglichen diesbezüglichen Einflussfaktoren auf der Datenbasis der "Studie zur Gesundheit von Kindern und Jugendlichen in Deutschland" (KiGGS) untersucht. In dieser bundesweiten Querschnittstudie wurden zwischen 2003 und 2006 17.641 null- bis 17-jährige Kinder und Jugendliche untersucht. Die Datenerfassung umfasste eine ärztliche Untersuchung des Kindes, ein Interview der Eltern und schriftliche Fragebögen. Neben Prävalenzschätzungen wurden multivariate logistische Regressionsanalysen durchgeführt. Zunehmendes Alter und männliches Geschlecht, eine atopische Vorerkrankung des Kindes, eine positive Familienanamnese für allergische Erkrankungen sowie ein niedriges Geburtsgewicht erhöhten das Risiko für Asthma bronchiale signifikant, ebenso Übergewicht und schimmlige Wände in der Wohnung. Das Stillen hatte keine schützende Wirkung. Ein niedriges Alter der Mutter bei der Geburt und Leben in ländlichen oder kleinstädtischen Regionen erwiesen sich als protektiv. Insgesamt zeigte sich, dass die genetische Prädisposition und eine atopische Vorerkrankung des Kindes die stärksten Risikofaktoren für Asthma bronchiale sind. Aber auch Umweltfaktoren (schimmlige Wände, Leben in ländlichen und kleinstädtischen Gemeinden) und einzelne Lebensstilfaktoren spielten eine modifizierende Rolle.

Schlüsselwörter

Asthma bronchiale $\cdot$ Risikofaktoren .

Gesundheitssurvey $\cdot$ Kinder · Jugendliche

\section{Determinants of asthma among children and adolescents in Germany. Results of the German Health and Examination Survey for Children and Adolescents (KiGGS)}

\section{Abstract}

In this study, associations between current asthma and possible determinants were studied using data of the German Health Interview and Examination Survey for Children and Adolescents ("Studie zur Gesundheit von Kindern und Jugendlichen in Deutschland," KiGGS). In this nationwide cross-sectional survey, 17,461 subjects aged $0-17$ years were examined between 2003 and 2006. Data collection included a medical examination of the child, an interview of the parents, and written questionnaires. Apart from prevalence estimates, multivariate logistic regression analyses were performed. Increasing age and male sex, previous atopic diseases of the child, a positive family history of allergic diseases and low birth weight were sig- nificant risk factors of asthma, as were overweight and moldy walls in the residence. Breast feeding was not associated with a reduced risk of asthma. Low age at delivery of the mother and living in rural or provincial regions were shown to be protected. Overall, this study suggests that allergies of the parents and previous atopic disease of the child are the strongest determinants of asthma. However, environmental factors (mold on walls, living in rural and provincial towns) and lifestyle factors could also modify asthma risk.

Keywords

Asthma - Risk factors · Health survey .

Children $\cdot$ Adolescents

\section{Prä- und perinatale Einflüsse}

Das Rauchen der Mutter in der Schwangerschaft und das Stillverhalten der Mutter hatten in der univariaten Auswertung keinen signifikanten Einfluss auf das Vorliegen einer Asthmaerkrankung. Bei Kindern mit einem Geburtsgewicht unter 2500 Gramm war die Er- krankungshäufigkeit geringfügig, jedoch nicht signifikant höher als bei Kindern mit einem höheren Geburtsgewicht. Auch ergaben sich keine signifikanten Unterschiede in der Asthmaprävalenz, wenn nach dem Alter der Mutter bei der Geburt geschichtet wurde. 
Tab. 1 Gewichtete Zwölf-Monats-Prävalenzen von Asthma bronchiale, geschichtet nach soziodemografischen Faktoren, Innenraum- und frühkindlichen Belastungen bei Kindern und Jugendlichen (KiGGS)

\begin{tabular}{|c|c|c|c|c|}
\hline \multirow[t]{2}{*}{ Einflussfaktor } & \multirow[t]{2}{*}{$\mathrm{N}^{\mathrm{a}}$} & \multicolumn{3}{|c|}{ 12-Monats-Prävalenz } \\
\hline & & $(\%)$ & $95 \%$ & idenzintervall \\
\hline \multicolumn{5}{|l|}{ Sozialer Status } \\
\hline Niedrig & 4619 & 3,6 & 3,0 & 4,3 \\
\hline Mittel & 7619 & 3,2 & 2,8 & 3,7 \\
\hline Hoch & 4545 & 3,2 & 2,7 & 3,8 \\
\hline \multicolumn{5}{|l|}{ Migrationshintergrund } \\
\hline Ja & 2945 & 2,8 & 2,2 & 3,5 \\
\hline Nein & 14.214 & 3,4 & 3,1 & 3,8 \\
\hline \multicolumn{5}{|l|}{ Region } \\
\hline Ost & 2843 & 3,5 & 2,9 & 4,3 \\
\hline West & 14.389 & 3,3 & 2,9 & 3,7 \\
\hline \multicolumn{5}{|l|}{ Gemeindegröße } \\
\hline Ländlich & 3083 & 2,8 & 2,2 & 3,6 \\
\hline Kleinstädtisch & 4726 & 2,9 & 2,4 & 3,4 \\
\hline Mittelstädtisch & 5024 & 3,4 & 2,8 & 4,1 \\
\hline Großstädtisch & 4400 & 4,0 & 3,4 & 4,8 \\
\hline \multicolumn{5}{|l|}{ Mindestens ein Elternteil Landwirt } \\
\hline $\mathrm{Ja}$ & 346 & 1,2 & 0,4 & 3,9 \\
\hline Nein & 16.886 & 3,4 & 3,0 & 3,7 \\
\hline \multicolumn{5}{|l|}{ Geschwisterzahl (leiblich) } \\
\hline Einzelkind & 3132 & 3,1 & 2,5 & 3,9 \\
\hline 1-3 Geschwister & 12.792 & 3,4 & 3,0 & 3,9 \\
\hline 4 und mehr Geschwister & 649 & 2,1 & 1,1 & 3,7 \\
\hline \multicolumn{5}{|l|}{ Anzahl älterer Geschwister im Haushalt } \\
\hline Keine & 8323 & 3,5 & 3,1 & 4,0 \\
\hline Eins & 5834 & 3,3 & 2,8 & 3,9 \\
\hline Mehr als eins & 2012 & 2,3 & 1,7 & 3,1 \\
\hline \multicolumn{5}{|l|}{ Vorschulische Betreuung } \\
\hline Ausschließlich in Familie & 4108 & 2,1 & 1,6 & 2,7 \\
\hline Familie + Kindereinrichtung & 12.708 & 3,7 & 3,3 & 4,1 \\
\hline \multicolumn{5}{|c|}{ Alter bei Betreuungsbeginn in einer Kindereinrichtung } \\
\hline Im ersten oder zweiten Lebensjahr & 2099 & 3,1 & 2,3 & 4,1 \\
\hline Im dritten oder vierten Lebensjahr & 7037 & 3,6 & 3,1 & 4,1 \\
\hline Im fünften Lebensjahr oder später & 2884 & 4,6 & 3,7 & 5,8 \\
\hline Ausschließlich zu Hause & 4108 & 2,1 & 1,6 & 2,7 \\
\hline \multicolumn{5}{|c|}{ Rauchen der Mutter während der Schwangerschaft } \\
\hline Regelmäßig & 821 & 4,3 & 3,0 & 6,2 \\
\hline Gelegentlich & 2086 & 3,7 & 2,9 & 4,7 \\
\hline Nie & 13.775 & 3,2 & 2,9 & 3,6 \\
\hline \multicolumn{5}{|l|}{ Alter der Mutter bei der Geburt } \\
\hline$<20$ Jahre & 420 & 2,0 & 0,9 & 4,0 \\
\hline 20-39 Jahre & 16.106 & 3,3 & 3,0 & 3,7 \\
\hline 40 + Jahre & 336 & 2,1 & 1,0 & 4,4 \\
\hline \multicolumn{5}{|l|}{ Geburtsgewicht (g) } \\
\hline$<2500$ & 944 & 4,4 & 3,1 & 6,3 \\
\hline 2500-3999 & 13.518 & 3,3 & 3,0 & 3,7 \\
\hline $4000+$ & 1951 & 2,8 & 2,1 & 3,7 \\
\hline \multicolumn{5}{|l|}{ Stillen } \\
\hline Jemals gestillt & 12.863 & 3,4 & 3,0 & 3,8 \\
\hline Nie gestillt & 3743 & 3,2 & 2,6 & 3,9 \\
\hline
\end{tabular}

\section{Innenraumbelastungen}

Von den untersuchten Innenraumbelastungen wurde nur für Schimmelpilzbefall ein Zusammenhang mit dem Auftreten von Asthma bronchiale in den letzten zwölf Monaten nachgewiesen. Kinder und Jugendliche, die in Wohnungen mit schimmligen Wänden in ihrem Aufenthaltsbereich lebten, waren häufiger an Asthma bronchiale erkrankt als Kinder und Jugendliche, die keinem Schimmelbefall ausgesetzt waren. Rauchen in der Wohnung sowie die Haltung von Haustieren im Allgemeinen hatten keinen Einfluss auf die Höhe der Zwölf-Monats-Prävalenz von Asthma bronchiale. In Bezug auf die Haltung einzelner Tierarten zeigte sich, dass die Asthmaprävalenz bei Kindern aus Familien, die Aquarien-/Terrarientiere hielten, signifikant höher war als bei Familien ohne solche Tierhaltung. Bei Familien mit Hundehaltung war die Prävalenz von Asthma bronchiale etwas geringer als bei Familien, die keine Hunde hielten.

\section{Atopische Vorerkrankungen und familiäre Vorbelastung}

Eine atopische Vorerkrankung des Kindes an Heuschupfen und/oder atopischem Ekzem sowie eine genetische Prädisposition hatten einen signifikanten Einfluss auf die Asthmaprävalenz (• Tab. 2). Kinder und Jugendliche, die bereits vorher an Heuschnupfen oder atopischem Ekzem erkrankt waren, waren 3,4-mal (Heuschnupfen) beziehungsweise 2,8-mal (atopisches Ekzem) so häufig von Asthma bronchiale betroffen wie Kinder und Jugendliche, die noch nie an diesen Erkrankungen gelitten hatten. Außerdem war die Asthmaprävalenz bei Kindern mit mindestens einem Elternteil, das an einer atopischen Erkrankung litt, doppelt so hoch wie bei Kindern, deren Eltern keine Allergiker waren (• Tab. 2). Dabei waren vor allem Kinder von Eltern, die selbst an Asthma bronchiale erkrankt waren, betroffen. Aber auch Kinder von Eltern mit Heuschnupfen hatten eine statistisch signifikante höhere Asthmaprävalenz. 
Tab. 1 Gewichtete Zwölf-Monats-Prävalenzen von Asthma bronchiale, geschichtet nach soziodemografischen Faktoren, Innenraum- und frühkindlichen Belastungen bei Kindern und Jugendlichen (KiGGS) (Fortsetzung)

\begin{tabular}{|c|c|c|c|c|}
\hline \multirow{3}{*}{$\begin{array}{l}\text { Einflussfaktor } \\
\text { Täglich }\end{array}$} & \multirow{3}{*}{$\begin{array}{l}\mathrm{N}^{\mathrm{a}} \\
2248\end{array}$} & \multicolumn{3}{|c|}{ 12-Monats-Prävalenz } \\
\hline & & \multirow{2}{*}{$\begin{array}{l}\text { (\%) } \\
3,5\end{array}$} & \multicolumn{2}{|c|}{ 95\%-Konfidenzintervall } \\
\hline & & & 2,7 & 4,6 \\
\hline Seltener als täglich & 2679 & 3,9 & 3,1 & 5,0 \\
\hline Nie & 11.814 & 3,1 & 2,7 & 3,6 \\
\hline \multicolumn{5}{|c|}{ Schimmlige Wände im Aufenthaltsbereich des Kindes } \\
\hline $\mathrm{Ja}$ & 861 & 5,4 & 4,0 & 7,3 \\
\hline Nein & 15.986 & 3,2 & 2,9 & 3,6 \\
\hline \multicolumn{5}{|l|}{ Haustierhaltung } \\
\hline $\mathrm{Ja}$ & 7734 & 3,7 & 3,2 & 4,2 \\
\hline Nein & 9143 & 3,0 & 2,6 & 3,5 \\
\hline \multicolumn{5}{|l|}{ Katze } \\
\hline $\mathrm{Ja}$ & 3075 & 3,1 & 2,5 & 3,8 \\
\hline Nein & 13.751 & 3,4 & 3,0 & 3,8 \\
\hline \multicolumn{5}{|l|}{ Hund } \\
\hline Ja & 2676 & 2,7 & 2,1 & 3,6 \\
\hline Nein & 14.150 & 3,4 & 3,1 & 3,8 \\
\hline \multicolumn{5}{|l|}{ Kleinsäuger } \\
\hline $\mathrm{Ja}$ & 2172 & 3,7 & 2,9 & 4,6 \\
\hline Nein & 14.654 & 3,3 & 2,9 & 3,7 \\
\hline \multicolumn{5}{|l|}{ Vögel } \\
\hline $\mathrm{Ja}$ & 1328 & 4,7 & 3,3 & 6,6 \\
\hline Nein & 15.498 & 3,2 & 2,9 & 3,5 \\
\hline \multicolumn{5}{|c|}{ Aquarien-/Terrarientiere } \\
\hline $\mathrm{Ja}$ & 1862 & 5,0 & 4,0 & 6,3 \\
\hline Nein & 14.964 & 3,1 & 2,8 & 3,5 \\
\hline
\end{tabular}

\section{Körpergewicht}

- Abb. 2 zeigt, dass die Zwölf-MonatsPrävalenz von Asthma bronchiale mit steigendem Body-Mass-Index zunahm. Kinder und Jugendliche mit Übergewicht oder Adipositas waren signifikant häufiger an Asthma bronchiale erkrankt als Kinder und Jugendliche mit normalem Körpergewicht.

\section{Multifaktorielle Auswertung}

In der multifaktoriellen Analyse (- Tab.3) erwiesen sich die Vorerkrankungen der Kinder an atopischem Ekzem oder an Heuschnupfen als die stärksten Einflussfaktoren auf die ZwölfMonats-Prävalenz (Heuschnupfen OR: 2,21, 95\%-KI: 1,65-2,96, atopisches Ekzem OR: 2,19, 95\%-KI: 1,83-3,03). Ebenso blieben der elterliche Allergiestatus, Übergewicht/Adipositas und Schimmelbefall im Aufenthaltsbereich des Kindes als signifikante Einflussgrößen erhalten. Im Unterschied zu den univariaten Analysen konnten im multiplen Modell ein niedriger sozialer Status (OR: 1,37, 95\%KI: 1,01-1,86) und ein geringes Geburtsgewicht $(<2500 \mathrm{~g})$ (OR: 1,84, 95\%-KI: 1,22-2,74) als signifikante Einflussgrößen identifiziert werden. Dagegen war der Einfluss der vorschulischen Betreuung ausschließlich innerhalb der Familie deutlich zurückgegangen. Das Merkmal „Stillen“ war im multiplen Modell ebenfalls ein statistisch signifikanter Einflussfaktor (OR: 1,38, 95\%-KI: 1,02-1,87). Signifikante Einflussfaktoren, die negativ (und damit protektiv) mit dem Auftreten einer Asthmaerkrankung zusammenhingen, waren im multiplen Modell die Gemeindegröße, das heißt Wohnen im ländlichen oder kleinstädtischen Raum, ein Alter der Mutter bei der Geburt von $<20$ Jahren und Hundehaltung in der Wohnung. Hingegen war die Haltung von Aquarien-/Terrarientieren positiv (risikoerhöhend) mit Asthma bronchiale assoziiert.

In einer altersstratifizierten Auswertung bei Kindern bis zehn Jahre und Kindern von elf bis 17 Jahren waren in beiden Altersgruppen positive Zusammenhänge zwischen Asthma bronchiale und elterlichem Allergiestatus sowie der kindlichen Vorerkrankung an Heuschnupfen oder atopischem Ekzem und Adipositas nachweisbar. Bei den älteren Kindern bestand zudem eine signifikant negative Assoziation zwischen Asthma bronchiale und einem starken Untergewicht. Bei den jüngeren Kindern waren Zugehörigkeit zum männlichen Geschlecht sowie eine Belastung durch schimmlige Wände im Aufenthaltsbereich des Kindes signifikant mit dem vermehrten Auftreten von Asthma bronchiale verbunden. Stillen und Haltung von Terrarien-/Aquarientieren waren nur bei den älteren Kindern positiv mit Asthma bronchiale assoziiert. Ein geringes Geburtsgewicht $(<2500 \mathrm{~g})$ ging in beiden Gruppen positiv mit einer Asthmaerkrankung einher, war jedoch nur bei den jüngeren Kindern statistisch signifikant. Ebenso war ein geringes Alter der Mutter bei der Geburt in beiden Altersgruppen negativ mit Asthma bronchiale assoziiert, jedoch nur bei den älteren Kindern statistisch signifikant.

Eine vertiefende Untersuchung des Einflusses des elterlichen Allergiestatus auf das Auftreten von Asthma bronchiale bei Kindern und Jugendlichen im multivariaten Modell zeigte, dass ein Asthma bronchiale bei Vater oder Mutter die Wahrscheinlichkeit für eine Asthmaerkrankung beim Kind fast um das Dreifache erhöhte ( $\bullet$ Abb. 3). Dabei spielte es keine Rolle, welches der beiden Elternteile an Asthma bronchiale litt. Eine Asthmaerkrankung beider Elternteile erhöhte ebenfalls die Wahrscheinlichkeit für eine kindliche Erkrankung, die jedoch wegen der geringen Fallzahl keine statistische Signifikanz erreichte. Im Vergleich zu Kindern von Asthmatikern war das Asthmarisiko bei Kindern von Eltern mit Heuschnupfen oder Neurodermitis deutlich geringer. Lediglich die mütterliche Erkrankung an Heuschnupfen erhöhte die Wahrscheinlichkeit einer Asthmaerkrankung beim Kind statistisch signifikant. 
Tab. 2 Gewichtete Zwölf-Monats-Prävalenzen von Asthma bronchiale bei Kindern und Jugendlichen (KiGGS), geschichtet nach Vorerkrankung der Probanden an Neurodermitis oder Heuschnupfen und nach Allergiestatus der Eltern

\begin{tabular}{|c|c|c|c|c|}
\hline \multirow[t]{2}{*}{ Einflussfaktor } & \multirow[t]{2}{*}{$\mathrm{N}^{\mathrm{a}}$} & \multicolumn{3}{|c|}{ 12-Monats-Prävalenz } \\
\hline & & (\%) & \multicolumn{2}{|c|}{ 95\%-Konfidenzintervall } \\
\hline Vorerkrankung beim Kind & & & & \\
\hline \multicolumn{5}{|c|}{ Neurodermitis (atopisches Ekzem) } \\
\hline $\mathrm{Ja}$ & 2172 & 7,6 & 6,3 & 9,0 \\
\hline Nein & 14.912 & 2,7 & 2,7 & 3,1 \\
\hline \multicolumn{5}{|l|}{ Heuschnupfen } \\
\hline Ja & 1592 & 9,2 & 7,7 & 11,1 \\
\hline Nein & 15.554 & 2,7 & 2,4 & 3,1 \\
\hline \multicolumn{5}{|c|}{ Elterliche Vorbelastung } \\
\hline \multicolumn{5}{|c|}{ Mindestens ein Elternteil mit einer atopischen/allergischen Erkrankung } \\
\hline $\mathrm{Ja}$ & 7194 & 4,5 & 4,1 & 5,1 \\
\hline Nein & 9242 & 2,3 & 1,9 & 2,7 \\
\hline \multicolumn{5}{|c|}{ Mindestens ein Elternteil Asthma bronchiale } \\
\hline Ja & 1397 & 8,9 & 7,3 & 10,8 \\
\hline Nein & 13.974 & 2,7 & 2,4 & 3,1 \\
\hline \multicolumn{5}{|c|}{ Mindestens ein Elternteil Heuschnupfen } \\
\hline Ja & 4997 & 4,5 & 3,9 & 5,3 \\
\hline Nein & 10.374 & 2,7 & 2,3 & 3,1 \\
\hline \multicolumn{5}{|c|}{ Mindestens ein Elternteil Neurodermitis (atopisches Ekzem) } \\
\hline $\mathrm{Ja}$ & 1397 & 4,3 & 3,3 & 5,6 \\
\hline Nein & 13.974 & 3,2 & 2,8 & 3,6 \\
\hline
\end{tabular}

Schichtet man im multivariaten Modell die Stichprobe der Kinder und Jugendlichen nach dem Allergiestatus der Eltern, so zeigte sich, dass sich bei Kindern und Jugendlichen mit einer positiven Familienanamnese (mindestens ein Elternteil hat einen positiven Allergiestatus) die Merkmale: männliches Geschlecht, Rauchen der Mutter während der Schwangerschaft, Stillen, niedriger sozialer Status, schimmlige Wände, die Haltung von Aquarien-/Terrarientieren sowie ein Geburtsgewicht $<2500 \mathrm{~g}$ als signifikante und risikoerhöhende Faktoren erwiesen. Dagegen waren Übergewicht und Adipositas sowie ein $\mathrm{Al}$ ter der Mutter bei der Geburt von jünger als 20 Jahren nur in der Schicht der Kinder und Jugendlichen mit negativer Familienanamnese statistisch signifikant mit einem erhöhten Asthmarisiko verbunden. In beiden Schichten war das Asthmarisiko signifikant verringert bei jüngeren Kindern (Altersgruppe < elf Jahre) und bei Kindern und Jugendlichen, die in einer ländlichen Umgebung oder im kleinstädtischen Umfeld lebten.

\section{Diskussion}

Obwohl Einflussfaktoren für Asthma bronchiale bereits in einzelnen Studienpopulationen in Deutschland untersucht wurden, ist dies die erste deutschlandweite Studie bei Kindern und Jugendlichen. Bei der Auswertung der KiGGS-Daten konnte eine Reihe von Einflussfaktoren auf eine bestehende Asthmaerkrankung, die in der internationalen Literatur beschrieben wurden, bestätigt werden. Hierzu zählen unter anderem der Atopiestatus des Kindes und der Eltern, frühkindliche Faktoren wie das Geburtsgewicht, Übergewicht und Adipositas sowie Schimmelbefall in der Wohnung.

Der im KiGGS beobachtete altersabhängige Zusammenhang zwischen Asthma bronchiale und Geschlecht, der zeigt, dass Asthma bronchiale bis zum 13. Lebensjahr häufiger bei Jungen und danach häufiger bei Mädchen vorkommt, wird in verschiedenen Berichten ebenfalls beschrieben $[9,15,16,17]$. Diese Häufigkeitsumkehr wird einerseits durch die bei Jungen kleineren bronchialen Querschnitte erklärt, die mit dem pubertären Wachs- tum stärker zunehmen als bei den Mädchen, sodass Männer im Allgemeinen größere bronchiale Querschnitte aufweisen als Frauen [15], andererseits können hormonelle Einflüsse $[15,17,18,19]$ oder geschlechtsspezifische Unterschiede in Umweltbelastungen $[15,17]$ eine Rolle spielen.

Von den im KiGGS untersuchten Innenraumbelastungen erwies sich nur das Vorhandensein eines Schimmelpilzbefalls im Aufenthaltsbereich des Kindes als signifikanter Risikofaktor für eine bestehende Asthmaerkrankung. Viele Studien, die in den letzten Jahren bei Erwachsenen und auch bei Kindern durchgeführt worden sind, haben gezeigt, dass übermäßige Innenraumfeuchte und Schimmel nachteilige Wirkungen auf die Gesundheit der Atemwege besitzen [20]. International wird eingeschätzt, dass eine ausreichende Evidenz für eine Assoziation zwischen Asthma(symptomen) und Schimmel beziehungsweise übermäßiger Innenraumfeuchte besteht $[21,22]$. Interessanterweise war der Zusammenhang zwischen einem Schimmelpilzbefall in der Wohnung und Asthma bronchiale nur bei den Kindern unter elf Jahren signifikant, während der Schimmelbefall bei den älteren Kindern keine signifikante Rolle zu spielen scheint. Ein vergleichbarer altersabhängiger Zusammenhang zwischen Asthma bronchiale und einem Schimmelpilzbefall im Schlafraum des Kindes wurde auch in den deutschen Zentren Münster und Greifswald im Rahmen der ISAACI-Studie beobachtet [4]. Möglicherweise erklärt sich diese Altersabhängigkeit dadurch, dass sich ältere Kinder seltener in der eigenen Wohnung aufhalten und deswegen geringer exponiert werden.

Der Einfluss der Tierhaltung auf die Asthmaprävalenz ist aufgrund des Querschnittdesigns der Studie schwer zu interpretieren. Das Halten von Fischen oder Terrarientieren bei Asthmatikern ist vermutlich nicht im Sinne einer Erhöhung des Erkrankungsrisikos zu verstehen, sondern wohl eher dadurch bedingt, dass durch das bewusste Meiden des potenziellen Risikofaktors "Haartiere“ ein „Umstieg“ auf „harmlosere“ Tiere erfolgte, um die Wünsche der Kinder nach Tiergefährten zu erfüllen. Dies zeigt sich an den Zahlen zur Häufigkeit der Hal- 


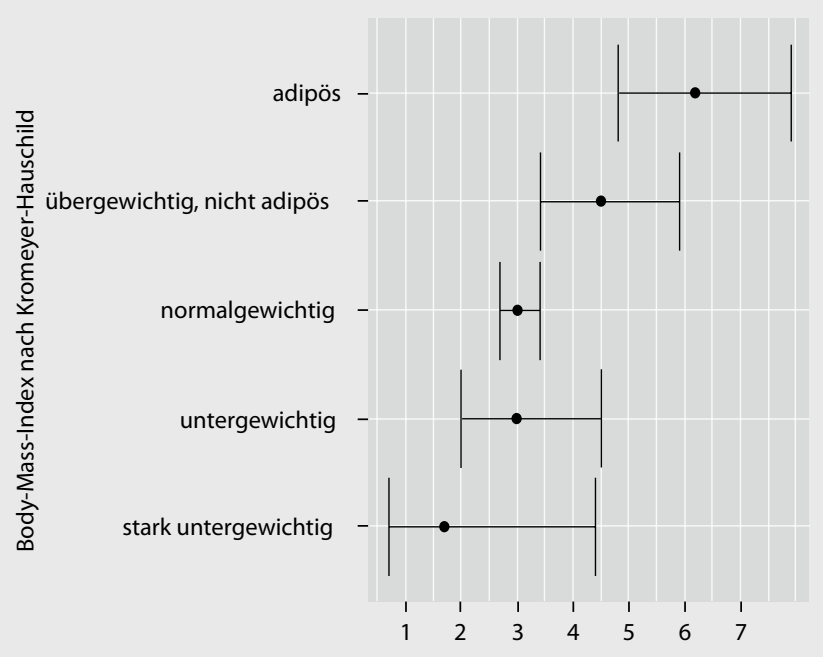

12-Monats-Prävalenz (\%) mit 95\%-Konfidenzintervall
Abb. $2<$ Zwölf-Monats-Prävalenz von Asthma bronchiale und Body-Mass-Index nach KromeyerHauschild (KiGGS, gewichtet)

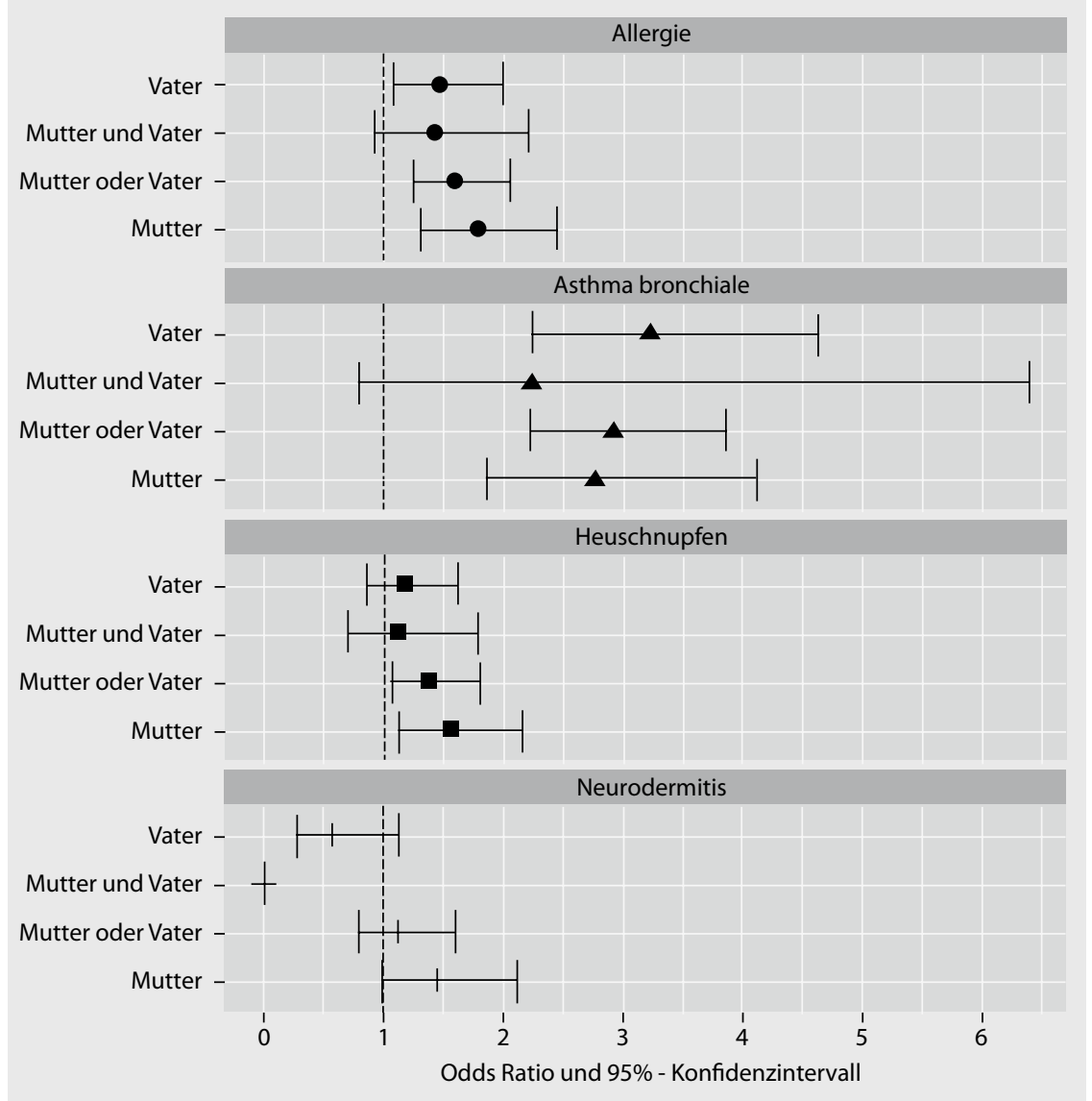

Abb. $3 \Delta$ Odds Ratios und 95\%-Konfidenzintervalle für die Assoziation von Asthma bronchiale und Allergiestatus der Eltern (KiGGS, gewichtet, adjustiert für alle im multivariaten Modell aufgenommenen Variablen)

tung dieser Tiere in den Haushalten der Probanden der KiGGS-Stichprobe. Insgesamt hatten 16,7\% der Kinder mit Asthma bronchiale zu Hause Aquarien/Terrarientiere. Bei Kindern ohne Asthmaerkrankung war die Prävalenz mit 10,9\% signifi- kant geringer. Ein ähnliches Verhalten der Probanden kann den vermeintlich schützenden Effekt der Hundehaltung erklären, da Familien mit an Asthma bronchiale erkrankten Kindern dazu neigen, seltener Hunde zu halten. Es ist jedoch auch mög- lich, dass hier ein wirklicher Effekt vorliegt, da bereits in früheren Studien ein protektiver Einfluss von Hundehaltung auf das Erkrankungsrisiko gezeigt wurde. Insgesamt ist die diesbezügliche Datenlage jedoch nicht konsistent [23].

Das Rauchen beziehungsweise Passivrauchen ist ein bedeutender Risikofaktor für Asthma bronchiale [4, 5, 9, 24]. Allerdings konnte im KiGGS das Rauchen in der Wohnung, die wichtigste Passivrauchbelastung bei Kindern, nicht mit einer bestehenden Erkrankung an Asthma bronchiale in Verbindung gebracht werden. Auch in einer Zusatzauswertung unter den elf- bis 17-jährigen Kindern hatten aktives Rauchen sowie der Aufenthalt in verrauchten Räumen keinen signifikanten Einfluss auf die Asthmaprävalenz. Die Daten zu Rauchverhalten und Passivrauchbelastung beruhen jedoch auf Selbstangaben der Eltern beziehungsweise der Kinder über zehn Jahre. Dies hat möglicherweise zur Untererfassung der Belastung durch Zigarettenrauch geführt, was eventuell das Fehlen eines Zusammenhangs zwischen Rauchbelastung und Asthma erklären könnte. Andererseits könnte auch hier in Kenntnis der Schädlichkeit des Rauchens eine verhaltensbedingte Gegensteuerung durch die Eltern von asthmatischen Kindern den fehlenden Zusammenhang erklären.

Eine Auswertung der Asthmaprävalenz nach der Gemeindegrößenklasse ergab, dass Kinder, die in ländlichen oder kleinstädtischen Regionen leben, deutlich seltener Asthma bronchiale hatten als Kinder aus Großstädten. Dies könnte mit der erhöhten Schadstoffbelastung in städtischen Gebieten zusammenhängen. Es ist jedoch nicht auszuschließen, dass auch andere mit der Wohnumgebung assoziierte Faktoren eine Rolle spielen.

Kinder, die auf Bauernhöfen mit Viehhaltung aufwuchsen, waren während ihrer Kindheit einem breiteren Spektrum an Keimen ausgesetzt, was in einer Reihe von Studien mit einem geringeren Asthmarisiko in Zusammenhang gebracht wurde [25, 26, 27]. Die vorliegende Auswertung der KiGGS-Daten zeigte zwar eine geringere Asthmaprävalenz sowie eine deutlich erniedrigte Odds Ratio bei Kindern, deren Väter oder Mütter von Beruf Landwirte waren, jedoch war die- 
Tab. 3 Ergebnisse der multivariaten logistischen Analyse mit Asthma bronchiale in den letzten zwölf Monaten als abhängiger Variable $(n=14.081)$

Einflussfaktor

Odds Ratio 95\%-Konfidenzintervall

\section{Altersgruppe}

Versus 14-17 Jahre

\begin{tabular}{llll}
\hline 0-2 Jahre & 0,16 & 0,08 & 0,32 \\
\hline 3-6 Jahre & 0,49 & 0,34 & 0,70 \\
\hline 7-10 Jahre & 0,73 & 0,55 & 0,97 \\
\hline 11-13 Jahre & 1,05 & 0,77 & 1,44 \\
\hline Geschlecht & 1,43 & 1,15 & 1,78
\end{tabular}

Jungen versus Mädchen

Sozialer Status

Versus hoch

\begin{tabular}{llcc}
\hline Niedrig & 1,37 & 1,01 & 1,86 \\
\hline Mittel & 1,07 & 0,83 & 1,39 \\
\hline $\begin{array}{l}\text { Migrantenstatus } \\
\text { Migrant versus Nicht-Migrant }\end{array}$ & 0,79 & 0,57 & 1,11 \\
\hline $\begin{array}{l}\text { Region } \\
\text { Ost- versus Westdeutschland }\end{array}$ & 1,00 & 0,77 & 1,31 \\
\hline $\begin{array}{l}\text { Vorschulische Betreuung ausschließlich in der Familie } \\
\text { J }\end{array}$ & 0,93 & 0,68 & 1,27
\end{tabular}

Ja versus nein

Anzahl älterer Geschwister im Haushalt

Versus zwei oder mehr

\begin{tabular}{llll}
\hline Keine & 1,21 & 0,81 & 1,82 \\
\hline Eins & 1,29 & 0,83 & 1,99 \\
\hline Mindestens ein Elternteil Landwirt & 0,36 & 0,09 & 1,50
\end{tabular}

Ja versus nein

Gemeindegröße

Versus großstädtisch

\begin{tabular}{llll}
\hline Ländlich & 0,67 & 0,49 & 0,93 \\
\hline Kleinstädtisch & 0,66 & 0,50 & 0,89 \\
\hline Mittelstädtisch & 0,85 & 0,63 & 1,13 \\
\hline $\begin{array}{l}\text { Rauchen der Mutter in der Schwangerschaft } \\
\text { Täglich oder gelegentlich versus nie }\end{array}$ & 1,32 & 0,96 & 1,81 \\
$\begin{array}{l}\text { Alter der Mutter bei der Geburt } \\
\text { Versus 20-39 Jahre }\end{array}$ & & & \\
\hline $\begin{array}{l}<20 \text { Jahre } \\
\text { 40+ Jahre }\end{array}$ & 0,23 & 0,07 & 0,67 \\
\hline $\begin{array}{l}\text { Geburtsgewicht (g) } \\
\text { Versus 2500-3999 g }\end{array}$ & 0,86 & 0,36 & 2,05 \\
\hline$<2500$ & & & \\
\hline $\begin{array}{l}\text { 4000+ } \\
\text { Stillen } \\
\text { Jemals gestillt versus nie }\end{array}$ & 1,84 & 1,22 & 2,74 \\
\hline
\end{tabular}

Body-Mass-Index nach Kromeyer-Hauschild

Versus normalgewichtig

\begin{tabular}{|c|c|c|c|}
\hline Stark untergewichtig & 0,64 & 0,23 & 1,75 \\
\hline Untergewichtig & 0,99 & 0,61 & 1,59 \\
\hline Übergewichtig, nicht adipös & 1,44 & 1,04 & 1,99 \\
\hline Adipös & 1,72 & 1,23 & 2,41 \\
\hline \multicolumn{4}{|l|}{$\begin{array}{l}\text { Rauchen in der Wohnung } \\
\text { Versus nie }\end{array}$} \\
\hline Täglich & 0,87 & 0,61 & 1,25 \\
\hline Seltener als täglich & 1,01 & 0,72 & 1,42 \\
\hline $\begin{array}{l}\text { Schimmlige Wände } \\
\text { Ja versus nein }\end{array}$ & 1,81 & 1,23 & 2,66 \\
\hline
\end{tabular}

ser Zusammenhang aufgrund zu geringer Fallzahlen in der Gruppe der "Landwirte“ statistisch nicht signifikant.

Der protektive Effekt eines frühzeitigen Kontakts mit Mikroorganismen (Hygiene-Hypothese), wurde zum ersten Mal 1989 diskutiert [28]. Im KiGGS wurden mehrere (früh)kindliche Belastungen unter diesem Aspekt untersucht, wobei jedoch keine schützende Wirkung vor Asthma bronchiale festgestellt wurde. Es wurde bereits an anderer Stelle darauf hingewiesen, dass ansteckende Krankheiten wie Keuchhusten, Masern und Mumps, bei den im KiGGS untersuchten Kindern und Jugendlichen keinen protektiven Effekt mit Blick auf Asthma bronchiale hatten [29]. Sonstige Faktoren, die mit einer erhöhten Exposition gegenüber Mikroorganismen einhergehen können, wie der Besuch einer Kindereinrichtung im Vorschulalter sowie die Zahl der älteren Geschwister im Haushalt, hatten in der vorliegenden Auswertung keinen wesentlichen Einfluss auf die Prävalenz von Asthma bronchiale oder erwiesen sich nicht als Risikofaktoren. Möglicherweise ist die mit dem frühzeitigen Besuch von Kindereinrichtungen beziehungsweise einer erhöhten Exposition gegenüber Krankheitserregern in der frühen Kindheit verbundene Minderung des Asthmarisikos nur zeitlich befristet. Darauf deuten Ergebnisse einer prospektiven Langzeitstudie aus den Niederlanden hin. In dieser Studie mit 3500 Kindern, die von der Geburt bis zum achten Lebensjahr untersucht wurden, hatten Kinder, die vor dem Schulanfang eine Kindertagesstätte besucht hatten, im Alter von fünf Jahren häufiger Atemwegsinfektionen und seltener Asthma bronchiale als Fünfjährige, die keine Kindereinrichtung besucht hatten. Im Alter von acht Jahren wurde kein Unterschied im Auftreten von Allergien und Asthma bronchiale zwischen beiden Gruppen beobachtet [30]. Über den Einfluss der Zahl an älteren Geschwistern auf das Asthmarisiko sind im Schrifttum unterschiedliche Ergebnisse zu finden. In einigen Studien war die Zahl der älteren Geschwister im Haushalt negativ mit dem Auftreten von Asthma bronchiale assoziiert $[9,28]$, in anderen war dies nicht der Fall [30]. 
Tab. 3 Ergebnisse der multivariaten logistischen Analyse mit Asthma bronchiale in den letzten zwölf Monaten als abhängiger Variable ( $n=14.081$ ) (Fortsetzung)

\begin{tabular}{lllc}
\hline Einflussfaktor & Odds Ratio & 95\%-Konfidenzintervall \\
\hline $\begin{array}{l}\text { Katzenhaltung } \\
\text { Ja versus nein }\end{array}$ & 0,94 & 0,70 & 1,26 \\
\hline $\begin{array}{l}\text { Hundehaltung } \\
\text { Ja versus nein }\end{array}$ & 0,69 & 0,51 & 0,93 \\
\hline $\begin{array}{l}\text { Kleinsäuger } \\
\text { Ja versus nein }\end{array}$ & 0,92 & 0,67 & 1,27 \\
\hline $\begin{array}{l}\text { Vögel } \\
\text { Ja versus nein }\end{array}$ & 1,28 & 0,87 & 1,89 \\
\hline $\begin{array}{l}\text { Aquarien-/Terrarientiere } \\
\text { Ja versus nein }\end{array}$ & 1,37 & 1,02 & 1,85 \\
\hline $\begin{array}{l}\text { Allergische Erkrankung bei den Eltern } \\
\text { Ja versus nein }\end{array}$ & 1,70 & 1,33 & 2,17 \\
\hline $\begin{array}{l}\text { Vorerkrankung Heuschnupfen } \\
\text { Ja versus nein }\end{array}$ & 2,21 & 1,65 & 2,96 \\
\hline $\begin{array}{l}\text { Vorerkrankung Neurodermitis, atopisches Ekzem } \\
\text { Ja versus nein }\end{array}$ & 2,36 & 1,83 & 3,03 \\
\hline
\end{tabular}

Stillen erwies sich im KiGGS als signifikanter Risikofaktor. Im internationalen Schrifttum finden sich bezüglich des Einflusses des Stillens auf das Asthma-bronchiale-Risiko differierende Ergebnisse. Es gibt sowohl Hinweise auf eine schützende als auch auf eine risikoerhöhende Wirkung des Stillens [9]. Die Stratifizierung nach dem Allergiestatus der Eltern zeigte allerdings, dass Stillen nur bei Kindern mit einer positiven $\mathrm{Fa}$ milienanamnese das Asthmarisiko erhöht und nicht bei Kindern mit einer negativen. Es handelt sich daher wahrscheinlich eher um einen scheinbaren Risikofaktor. Die höhere OR erklärt sich dadurch, dass Eltern mit einem positiven Allergiestatus die Stillempfehlungen häufiger umsetzen als Eltern ohne Allergien. Kinder von leiblichen Eltern mit Allergien wurden signifikant häufiger gestillt als Kinder von Eltern ohne Allergien (mit Allergien: 79,3\%, 95\%KI 77,5\%-80,9\% versus ohne Allergien $74,3 \%$, 95\%-KI 72,6\%-76,0\%). Auch in der altersstratifizierten Auswertung fanden sich unterschiedliche Ergebnisse, wobei Stillen das Asthmarisiko nur bei den elf- bis 17-jährigen, jedoch nicht bei den null- bis zehnjährigen Kindern signifikant erhöhte. Dies könnte auf einen Erinnerungsbias hinweisen. Es ist unumstritten, dass Stillen zahlreiche positive Gesundheitseffekte, darunter auch eine protektive Wirkung vor Infektionskrankheiten haben kann [31, 32]. Daher sollte das Ergebnis dieser Studie nicht im Sinne eines Stillverzichts interpretiert werden.

Das Rauchen der Mutter während der Schwangerschaft wirkt sich negativ auf die vorgeburtliche Entwicklung der Lunge aus. Hierdurch kann die Lungenfunktion nach der Geburt und in den ersten Lebensjahren verringert sein, was auch zu negativen Folgen in späteren Lebensabschnitten führen kann $[33,34]$. In verschiedenen Studien zeigte sich eine positive Beziehung zwischen mütterlichem Rauchen in der Schwangerschaft und dem Erkrankungsrisiko für Asthma bronchiale während der Kindheit $[35,36]$. In der vorliegenden Auswertung war das Rauchen der Mutter während der Schwangerschaft in der Gesamtstichprobe nicht signifikant mit dem Asthmarisiko des Kindes assoziiert. Dieser Befund hängt möglicherweise damit zusammen, dass im multivariaten Modell sowohl das Geburtsgewicht als auch das Rauchen während der Schwangerschaft berücksichtigt wurden. Ein niedriges Geburtsgewicht ist mit einem erhöhten Asthmarisiko verbunden, wie in dieser Auswertung und durch eine Vielzahl anderer Studien [36, 37, 38] gezeigt werden konnte. Da Rauchen in der Schwangerschaft positiv mit niedrigem Geburtsgewicht assoziiert ist, ist nicht auszuschließen, dass bei gleichzeitiger Berücksichtigung beider Merkmale in der Analyse der Zusammenhang zwischen Rauchen in der Schwangerschaft und Asthma bronchiale maskiert wird.
Wird jedoch die Familienanamnese für Allergien berücksichtigt, so zeigt sich bei Kindern und Jugendlichen mit einer positiven Familienanamnese ein statistisch signifikant erhöhtes Asthmarisiko im Zusammenhang mit Rauchen der Mutter während der Schwangerschaft.

Der beobachtete risikoerhöhende Effekt von Übergewicht und insbesondere von Adipositas auf Asthma bronchiale wurde wiederholt berichtet [39, 40, 41]. Verschiedene Studien haben gezeigt, dass ein hoher BMI mit einer verringerten Lungenfunktion beim Kind einhergeht $[40,41]$ und dass eine Gewichtsreduzierung bei adipösen Asthmatikern zu einer Verbesserung der Lungenfunktion und einer Verringerung der Asthmasymptome führen kann [40]. Dies deutet darauf hin, dass Lebensstilfaktoren wie mangelnde körperliche Aktivität oder übermäßiger Lebensmittelverzehr, die bekanntlich zu Übergewicht beziehungsweise Adipositas führen, eine Rolle spielen können. Der Zusammenhang zwischen Übergewicht/Adipositas und Asthma wurde insbesondere bei Kindern und Jugendlichen mit einer negativen Familienanamnese für Allergien und nicht bei Kindern mit einer positiven Familienanamnese beobachtet.

Zusammenfassend zeigt die vorliegende Auswertung der KiGGS-Daten, dass sowohl eine familiäre atopische Vorbelastung als auch eine atopische Vorerkrankung des Kindes die vergleichsweise stärksten Determinanten für Asthma bronchiale sind, aber auch bestimmte Umweltfaktoren wie Schimmel in der Wohnung und einige Lebensstilfaktoren eine Rolle als Risikofaktoren spielen. Ebenso ergaben sich Hinweise auf einen protektiven Einfluss des Wohnumfeldes (Wohnen im ländlichen Raum). Kritisch ist anzumerken, dass in Querschnittstudien eine Erfassung von Exposition und Wirkung im gleichen Zeitrahmen erfolgt, was bei Zusammenhangsanalysen zur Verzerrung von Risikoschätzungen führen kann, insbesondere dann, wenn die Exposition zeitlich nicht konstant bleibt, sondern durch Verhaltensänderungen der Probanden eine Modifikation erfährt. Darüber hinaus sind aufgrund des Querschnittdesigns dieser Studie Aussagen zur Kausalität der Beziehungen nicht mög- 
lich. Aus diesen Gründen sind zusätzlich Längsschnittstudien erforderlich, um Vermutungen über die Kausalität der beobachteten Zusammenhänge zu bestätigen.

\section{Korrespondenzadresse}

\section{Laußmann}

Abteilung Epidemiologie und

Gesundheitsberichterstattung,

Fachgebiet Epidemiologie nicht übertragbarer

Erkrankungen, Robert Koch-Institut

General-Pape-Str. 62-66, 12101 Berlin

laussmannd@rki.de

Danksagung. Wir danken allen Kindern und deren Eltern, die am deutschen Kinder- und Jugendgesundheitssurvey teilgenommen haben, für ihre Mitarbeit.

Interessenkonflikt. Der korrespondierende Autor gibt an, dass kein Interessenkonflikt besteht.

\section{Literatur}

1. Buhl R, Berdel D, Criee CP et al (2006) Guidelines for diagnosis and treatment of asthma patients. Pneumologie 60:139-177

2. Bundesärztekammer, Kassenärztliche Bundesvereinigung, Arbeitsgemeinschaft der Wissenschaftlichen Medizinischen Fachgesellschaften (AWMF) (Hrsg) (2010) Nationale VersorgungsLeitlinie Asthma Kurzfassung. http://www.asthma.versorgungsleitlinien.de

3. ISAAC (1998) Worldwide variation in prevalence of symptoms of asthma, allergic rhinoconjunctivitis, and atopic eczema: ISAAC. The International Study of Asthma and Allergies in Childhood (ISAAC) Steering Committee. Lancet 351:1225-1232

4. Duhme H, Weiland SK, Rudolph P et al (1998) Asthma and allergies among children in West and East Germany: a comparison between Munster and Greifswald using the ISAAC phase I protocol. International Study of Asthma and Allergies in Childhood. Eur Respir J 11:840-847

5. Eder W, Ege MJ, Mutius E von (2006) The asthma epidemic. N Engl J Med 355:2226-2235

6. Pearce N, Ait-Khaled N, Beasley R et al (2007) Worldwide trends in the prevalence of asthma symptoms: phase III of the International Study of Asthma and Allergies in Childhood (ISAAC). Thorax 62:758-766

7. Mutius E von (2000) The burden of childhood asthma. Arch Dis Child 82(Suppl 2):II2-II5

8. Schramm B, Ehlken B, Smala A et al (2003) Cost of illness of atopic asthma and seasonal allergic rhinitis in Germany: 1-yr retrospective study. Eur Respir J 21:116-122

9. Subbarao P, Mandhane PJ, Sears MR (2009) Asthma: epidemiology, etiology and risk factors. CMAJ 181:E181-E190

10. Kurth BM, Kamtsiuris $\mathrm{P}$, Holling $\mathrm{H}$ et al (2008) The challenge of comprehensively mapping children's health in a nation-wide health survey: design of the German KiGGS-Study. BMC Public Health 8:196
11. Kurth BM (2007) The German Health Interview and Examination Survey for Children and Adolescents (KiGGS): an overview of its planning, implementation and results taking into account aspects of quality management. Bundesgesundheitsbl Gesundheitsforsch Gesundheitsschutz 50:533-546

12. Kamtsiuris $P$, Lange $M$, Schaffrath Rosario $A$ (2007) The German Health Interview and Examination Survey for Children and Adolescents (KiGGS): sample design, response and nonresponse analysis. Bundesgesundheitsbl Gesundheitsforsch Gesundheitsschutz 50:547-556

13. Winkler J, Stolzenberg H (1999) Social class index in the Federal Health Survey. Gesundheitswesen 61:S178-S183

14. Kromeyer-Hauschild K, Wabitz M (2001) Perzentile für den Body-Mass-Index für das Kindes- und Jugendalter unter Heranziehung verschiedener deutscher Stichproben. Monatsschr Kinderheilkd 149:807-818

15. Becklake MR, Kauffmann F (1999) Gender differences in airway behaviour over the human life span. Thorax 54:1119-1138

16. Arbes SJ, Guo X, Orelien J, Zeldin DC (2004) Interaction between sex and age in the prevalence of current asthma. J Allergy Clin Immunol 113:S302

17. Almqvist C, Worm M, Leynaert B (2008) Impact of gender on asthma in childhood and adolescence: a GA2LEN review. Allergy 63:47-57

18. Jensen-Jarolim E, Untersmayr E (2008) Gender-medicine aspects in allergology. Allergy 63:610-615

19. Carey MA, Card JW, Voltz JW et al (2007) It's all about sex: male-female differences in lung development and disease. Trend Endocrinol Metab 18:308-313

20. Fisk WJ, Lei-Gomez Q, Mendell MJ (2007) Metaanalyses of the associations of respiratory health effects with dampness and mold in homes. Indoor Air 17:284-296

21. IOM (2004) Damp indoor spaces and health, institute of medicine. National Academy Press, Washington, DC

22. WHO (2006) Report on the WHO technical meeting on quatifying disease from inadequate housing. Copenhagen, Denmark

23. Chen CM, Tischer C, Schnappinger $M$, Heinrich J (2010) The role of cats and dogs in asthma and allergy - a systematic review. Int J Hyg Environ Health 213:1-31

24. Strachan DP, Cook DG (1998) Health effects of passive smoking. 6. Parental smoking and childhood asthma: longitudinal and case-control studies. Thorax 53:204-212

25. Mutius E von (2007) Asthma and allergies in rural areas of Europe. Proc Am Thorac Soc 4:212-216

26. Ege MJ, Mayer M, Normand AC et al (2011) Exposure to environmental microorganisms and childhood asthma. N Engl J Med 364:701-709

27. Riedler J, Braun-Fahrlander C, Eder W et al (2001) Exposure to farming in early life and development of asthma and allergy: a cross-sectional survey. Lancet 358:1129-1133

28. Strachan DP (1989) Hay fever, hygiene, and household size. BMJ 299:1259-1260

29. Langen U, Röhmel J (2008) Correlations between allergic and infectious diseases - results of the latest German National Health Survey (NHS98) and the German health interview and examination survey for children and adolescents (KiGGS). Open Allergy J 1:1-7
30. Caudri D, Wijga A, Scholtens S et al (2009) Early daycare is associated with an increase in airway symptoms in early childhood but is no protection against asthma or atopy at 8 years. Am J Respir Crit Care Med 180:491-498

31. Horta BL, Bahl R, Martines JC, Victora CG (2007) Evidence on the long-term effects of breastfeeding: systematic review and meta-analyses. World Health Organisation, Genf

32. WHO (2002) The optimal duration of exclusive breastfeeding. Report of an expert consultation. World Health Organisation, Genf

33. Hayatbakhsh MR, Sadasivam S, Mamun AA et al (2009) Maternal smoking during and after pregnancy and lung function in early adulthood: a prospective study. Thorax 64:810-814

34. Moshammer H, Hoek G, Luttmann-Gibson $\mathrm{H}$ et al (2006) Parental smoking and lung function in children: an international study. Am J Respir Crit Care Med 173:1255-1263

35. Haberg SE, Stigum H, Nystad W, Nafstad P (2007) Effects of pre- and postnatal exposure to parental smoking on early childhood respiratory health. Am J Epidemiol 166:679-686

36. Jaakkola JJ, Gissler M (2004) Maternal smoking in pregnancy, fetal development, and childhood asthma. Am J Public Health 94:136-140

37. Brooks AM, Byrd RS, Weitzman M et al (2001) Impact of low birth weight on early childhood asthma in the United States. Arch Pediatr Adolesc Med 155:401-406

38. Nepomnyaschy L, Reichman NE (2006) Low birthweight and asthma among young urban children. Am J Public Health 96:1604-1610

39. Noal RB, Menezes AM, Macedo SE, Dumith SC (2011) Childhood body mass index and risk of asthma in adolescence: a systematic review. Obes Rev 12:93-104

40. Sin DD, Sutherland ER (2008) Obesity and the lung: 4. Obesity and asthma. Thorax 63:10181023

41. Spathopoulos D, Paraskakis E, Trypsianis G et al (2009) The effect of obesity on pulmonary lung function of school aged children in Greece. Pediatr Pulmonol 44:273-280 